\title{
PROCESO DE INCLUSIÓN DE ESTUDIANTES SORDOS EN INSTITUCIONES EDUCATIVAS PÚBLICAS
}

\section{PROCESS FOR INCLUSION OF DEAF STUDENTS IN PUBLIC EDUCATIONAL INSTITUTIONS}

\section{VERÓNICA MALDONADO ${ }^{1}$}

LUCY RÍOS ${ }^{2}$

\section{ELKING ARAUJO ${ }^{3}$}

Recibido: 18 de junio de 2018 Aceptado: 22 de octubre de 2018

\footnotetext{
${ }^{1}$ Pontificia Universidad Católica del Ecuador, Facultad de Psicología, Quito, Ecuador. vmaldonado794@puce.edu.ec

${ }^{2}$ Pontificia Universidad Católica del Ecuador, Facultad de Psicología, Quito, Ecuador. lucyldu777@gmail.com

${ }_{3}^{3}$ Pontificia Universidad Católica del Ecuador, Facultad de Psicología, Quito, Ecuador eraraujo@puce.edu.ec
} 
ill|lin 102 


\section{PROCESO DE INCLUSIÓN DE ESTUDIANTES SORDOS EN INSTITUCIONES EDUCATIVAS PÚBLICAS}

\section{PROCESS FOR INCLUSION OF DEAF STUDENTS IN PUBLIC EDUCATIONAL INSTITUTIONS}

Verónica Maldonado, Lucy Ríos, Elking Araujo.

Palabras claves: Inclusión, Educación, sordera, necesidades educativas especiales

Keywords: Inclusion, Education, deafness, special educational needs

\section{RESUMEN}

Las políticas públicas del Estado ecuatoriano, en materia de inclusión educativa, han motivado una transformación en la constitución y gestión de las instituciones educativas. La inclusión educativa no siempre encuentra las condiciones adecuadas que le permitan ser efectiva y eficaz. Este artículo informa de la investigación y sus resultados acerca de las dificultades que encuentra el pro- ceso de inclusión de estudiantes sordos en instituciones educativas de carácter público. Para ello, se seleccionaron cinco áreas en las que se indagó la existencia de dificultades de inclusión -equidad, sensibilización, inclusión social, recursos educativos y capacitación-, así como sus características. Para la muestra se escogieron representantes de toda la comunidad educativa: estudiantes sordos, 
docentes, estudiantes sin discapacidad, autoridades y cabezas de familia (tanto de estudiantes sordos como de los que no la tienen). Los instrumentos utilizados fueron la entrevista y la encuesta. El análisis de los resultados, de tipo cualitativo, busca definir las áreas en las que debe fortalecerse este proceso de inclusión de cara a la consecución del ideal constitucional de un Ecuador con igualdad de oportunidades.

\section{ABSTRACT}

The public policies of the Ecuadorian State in matters of educational inclusion have motivated a transformation in the constitution and management of educational institutions. Educational inclusion does not always to the proper conditions for effectiveness. This article reports on the research and results regarding the difficulties encountered in the inclusion process for deaf students in public educational institutions. Five areas were thus selected, in which the existence of inclusion difficulties -equity, sensitization, social inclusion, educatio- nal resources and training- was investigated, as well as their characteristics. For the sample, representatives from the educational community were chosen: deaf students, teachers, students without disabilities, authorities and heads of families (both deaf and non-deaf students). The instruments used were the interview and the survey. The qualitative analysis of the results seeks to define the areas in which this inclusion process should be strengthened in order to achieve the constitutional ideal of an Equal Opportunity Ecuador.

\section{INTRODUCCIÓN}

Los nuevos marcos legales y los reglamentos que determinan el quehacer educativo en el Ecuador desde finales de la primera década del siglo XXI han promovido, en un real ajuste de cuentas con la tendencia actual, un proceso de inclusión educativa. Este ámbito, y las dificultades que en la práctica ha encontrado su ejecución, han llamado nuestro interés investigador. Partimos de concebir a la inclusión educativa como un interés por practicar un aprendizaje en el que primen por igual la calidad y la exigencia así como las capacidades 
de cada estudiante (Echeita, 2008, pág. 11). Dicho así, la práctica demuestra que el reto educativo se agiganta y las dificultades se multiplican. ¿Cuáles son, entonces, las mayores dificultades que surgen en los procesos de inclusión educativa? Los contextos educativos marcan las diferencias y esto nos lleva a circunscribir esta interrogante a un medio educativo específico: tomamos contacto con una institución educativa de carácter público-estatal.

A fin de contestar a nuestro problema de investigación, establecimos cinco áreas en las cuales indagar esas dificultades. Dichas áreas se convirtieron en nuestros objetivos secundarios: a) analizar las dificultades de inclusión en cuanto a la equidad de trato de la comunidad educativa hacia los estudiantes sordos; b) verificar la sensibilidad de la comunidad educativa frente al proceso de inclusión de los estudiantes sordos; c) describir las limitaciones del proceso de inclusión social en el ámbito educativo de los estudiantes sordos; d) especificar los recursos educativos valorados por los docentes como de mejor impacto para el proceso de inclusión educativa de los estudiantes sordos; $y, e$ ) indicar las principales necesidades de capacitación en materia de inclusión educativa que presenta el personal docente y psicoeducativo implicado en la inclusión de los estudiantes sordos.
Fueron cinco las áreas en las que indagamos los problemas de la inclusión educativa: equidad, sensibilidad, inclusión social, recursos educativos y capacitación. La equidad corresponde con la obligación de brindar una educación que ayude más a quien más lo requiere a fin de que todos los educandos lleguen a la misma meta educativa (López Cortiñas, 2005, p. 10). En este contexto se debe tomar en cuenta el principio de individualización, el cual hace referencia a la consideración de los individuos como seres únicos e irrepetibles. Por tanto, para lograr una meta educativa se debe considerar como eje principal las necesidades de cada uno de los educandos.

Entendemos por sensibilidad la asunción de actitudes positivas en las que priman valores positivos de respeto, solidaridad, valoración y tolerancia ante la discapacidad (Ministerio de Educación del Ecuador, 2011, p. 3). Por supuesto, la sensibilidad se adquiere a partir de un proceso de similar nombre en el que diversos fenómenos provocan cambios en las posturas idiosincráticas de las personas. Precisamente la ausencia de conocimiento acerca de las necesidades educativas especiales es uno de los fundamentos de la ausencia de sensibilidad hacia su tratamiento pedagógico.

En cambio, la inclusión social tiene que ver con la integración de los ciudadanos en las distintas agrupaciones 
que componen la sociedad a la que pertenecen (Boza Carreño, Méndez Garrido, Monescillo Palomo, \& Toscano Cruz, 2010, p. 113). Los procesos de inclusión social deben ser conducidos a acortar la brecha de desigualdad, a través del trabajo con énfasis en los grupos de atención prioritaria y personas en situación de vulnerabilidad.

Los recursos educativos pueden ser vistos de una forma más amplia, sin limitarse a considerarlos como meros recursos materiales ni como únicos vehículos de información; más que ello, son elementos que tornan más fácil la experiencia de aprendizaje (Kaplún, 2005, p. 143). Estos recursos deben constituirse en un verdadero potencial dentro de los procesos de enseñanza y aprendizaje. Su uso adecuado o inadecuado podría propiciar un resultado de aprendizaje significativo o un fracaso en el proceso. Los recursos educativos deben tener una intencionalidad definida previamente en la planificación de los procesos llevados a cabo en el nivel de los contextos educativos.

Finalmente, la capacitación se comprende como la capacidad de interacción de los individuos con el contexto, es decir, con las circunstancias que rodean la acción. En este caso, el aprendizaje, esto es la institución, en cuanto lugar, pero también en cuanto a los grupos humanos que la conforman: autoridades, estudiantes, docentes y padres de familia (Marcelo García \& Vaillant, 2009, p. 77). Las capacitaciones en educación deben partir de las necesidades y la realidad de cada contexto con el fin de mejorar la calidad educativa.

Nos acercamos a la indagación de los problemas de inclusión educativa en estas áreas, a través de un enfoque metodológico psicológico-humanista. Desde esta óptica, asumimos como fundamento el valor del ser humano y su potencialidad, visión estrechamente relacionada con el tema de la discapacidad, que determina como prioritaria la aceptación incondicional, el respeto de los derechos y la satisfacción de las necesidades de cada persona en busca de su desarrollo personal y su autorrealización. Los diversos instrumentos metodológicos son de tipo mixto, puesto que su estudio se realizó por medio de interpretación de variables cuantitativas y cualitativas; y, también de tipo transversal por haberse realizado en un punto específico del tiempo. Adicionalmente se destaca la utilización de técnicas tradicionales de gran valía como las encuestas, entrevistas, talleres, investigaciones de campo a personas que se encuentran directamente involucradas con este tema, dentro del entorno que se deja especificado en líneas anteriores. 


\section{MÉTODO}

\section{Características de la población}

La población de la institución está formada por 1.800 estudiantes, 95 docentes y 3 miembros del Departamento de Consejería Estudiantil (DECE). La muestra, a su vez, está compuesta por los siguientes grupos: a) 13 estudiantes sordos -con edades de 14 a 21 añosque se encuentran incluidos en educación regular, y que cursaban niveles desde octavo año de Educación Básica Superior hasta primer año de Bachillerato General Unificado; el 53\% son hombres y el $47 \%$ de estudiantes son mujeres; b) 119 estudiantes oyentes, compañeros de clase, de los cuales el 60\% son hombres y el 40\% mujeres; c) 21 docentes que incluyen a las profesoras intérpretes de lengua de señas que laboran en los respectivos paralelos en los que se desarrollaba el programa de inclusión educativa durante el año 2017; el 71\% son docentes mujeres y el 29\% docentes hombres; d) 12 padres de familia de los estudiantes sordos; el $75 \%$ son madres y el 25\% padres y; e) 3 profesionales del Departamento de Consejería Estudiantil (DECE), todas mujeres. Los participantes son de nacionalidad ecuatoriana, de la región Sierra Centro del país.

\section{Normas éticas de investigación}

Se firmaron consentimientos in- formados con los docentes, padres de familia y personal del DECE, de igual manera con los estudiantes sordos mayores de edad y, en el caso de los estudiantes menores de edad, el consentimiento informado fue firmado por su representante legal, para lo cual se tomó en cuenta previamente el asentimiento del mismo menor de edad.

\section{Instrumentos}

Los instrumentos que se emplearon como parte del trabajo de campo de la presente investigación dentro de la institución fueron entrevistas semiestructuradas para autoridades, miembros del DECE y los estudiantes sordos con el fin de recolectar información sobre sus historias de vida. También se aplicaron encuestas de preguntas cerradas y abiertas a toda la población objeto de este trabajo investigativo.

Con las entrevistas y las encuestas indagamos los problemas de la inclusión educativa: equidad, sensibilidad, inclusión social, recursos educativos y capacitación.

En el caso de los docentes se les consultó acerca del nivel de aceptación que tenían hacia los procesos de inclusión, las capacitaciones y las jornadas de sensibilización recibidas, así como los recursos educativos y la percepción en 
la relación entre los estudiantes sordos y los estudiantes oyentes.

A los estudiantes sordos se les preguntó acerca de su inclusión en el colegio, su relación con los docentes y con sus compañeros oyentes, también se les consultó sobre el tema de la discriminación y el nivel de satisfacción frente a las necesidades académicas, sociales, materiales y de accesibilidad.

A los estudiantes oyentes se les consultó sobre la aceptación a los procesos de inclusión desde su percepción como estudiantes. Además, brindaron información acerca de su relación con sus compañeros sordos, los espacios y tiempos compartidos dentro de las actividades académicas y extracurriculares. Se les consultó su parecer acerca de la posible interferencia que podría evidenciarse debido a la inclusión de estudiantes sordos en un aula de clases regular.

Los padres de familia brindaron información acerca del avance académico y social de sus hijos posteriores al proceso de inclusión, además opinaron acerca de las relaciones interpersonales de sus hijos y las necesidades presentadas.

Al personal del DECE se le consultó sobre los métodos que llevaban a cabo en la institución con miras a que el proceso fuera eficaz. Brindaron información acerca de los temas de capacitación, sensibilización y participación de los estudiantes sordos en la institución.

En las entrevistas personalizadas a los estudiantes sordos se abordaron temas de importancia. Por ejemplo, se les consultó acerca de sus datos personales, historia escolar desde su primer proceso de inclusión, sus relaciones familiares y sociales. Se enfatizó en las necesidades y demandas dentro de su inclusión educativa con respecto a los aspectos académicos, sociales, recursos, adaptaciones de comunicación, adaptaciones curriculares y otros.

La aplicación de las encuestas y entrevistas fue supervisada por una profesional del DECE de la institución y responsable del proceso de inclusión educativa. Estos instrumentos fueron validados por profesionales de la Psicología Educativa a través de revisiones consecutivas.

\section{Técnicas de análisis de datos}

Al realizar la fase de análisis -O diagnóstico de necesidades- se efectuaron entrevistas a los estudiantes sordos para conocer sus historias de vida, así como también a los especialistas encargados del proceso de inclusión educativa de la institución educativa seleccionada, para el respectivo análisis de este punto al correlacionarlas con las encuestas aplicadas previamente. Las entrevistas fueron grabadas con el consentimiento de 
los participantes, a quienes, además, se les comunicó que las historias de vida no serían expuestas o evidenciadas en este trabajo textualmente.

\section{Procedimiento}

El proceso de investigación inició con un primer contacto con las autoridades de la institución para realizar la propuesta. Se logró la aceptación y se procedió a solicitar la autorización al distrito del Ministerio de Educación al que corresponde la institución educativa de estudio.

La institución de estudio es de carácter educativo fiscal y está ubicada en la Sierra ecuatoriana. Oferta los niveles de Básica Superior (octavo, noveno, décimo año) y Bachillerato General Unificado (primero, segundo, tercero). Además, brinda la oportunidad de una formación en las especialidades de Contabilidad e Informática. Asimismo, cuenta con un programa de Bachillerato Internacional.

Una de las actividades trascendentales que se está efectuando es la inclusión de estudiantes sordos en la educación regular, siendo esta institución una de las primeras en incursionar en la atención a la diversidad. Esta actividad se desarrolla en cumplimiento a lo dispuesto en el Acuerdo Ministerial 295-13 promulgado en el año 2013 por el Ministerio de Educación del Ecuador.

\section{RESULTADOS}

Presentamos aquí los resultados de la investigación clasificados según las cinco grandes áreas que detallamos con anterioridad. En cada área se presenta la pregunta de la encuesta aplicada, a qué grupo responde y los resultados porcentuales de esas respuestas.

\section{Área: EQUIDAD}

\section{Responden: Estudiantes sordos}

¿Durante su permanencia en el colegio ha sentido algún tipo de discriminación hacia usted o a sus compañeros sordos?

\begin{tabular}{|l|l|}
\hline SI & $85 \%$ \\
\hline NO & $15 \%$ \\
\hline
\end{tabular}




\begin{tabular}{|l|c|c|}
\hline Opciones & Frecuencia & Porcentaje \\
\hline Agresión física & 0 & $0 \%$ \\
\hline Agresión verbal & 2 & $15 \%$ \\
\hline Comentario ofensivo & 0 & $0 \%$ \\
\hline Falta de atención en el bar de la institución & 3 & $23 \%$ \\
\hline Los profesores no prestan atención a sus necesidades & 4 & $31 \%$ \\
\hline No responde & 4 & $31 \%$ \\
\hline
\end{tabular}

En caso de que haya evidenciado parte de quién ha ocurrido: algún tipo de discriminación, indique por

\begin{tabular}{|l|c|c|}
\hline Opciones & Frecuencia & Porcentaje \\
\hline De sus compañeros oyentes & 4 & $31 \%$ \\
\hline De sus maestros & 8 & $62 \%$ \\
\hline De las autoridades & 4 & $31 \%$ \\
\hline De los padres de familia & 3 & $23 \%$ \\
\hline Ninguno, me parece que todos han sido respetuosos & 1 & $8 \%$ \\
\hline
\end{tabular}

Responden: Estudiantes oyentes conducta discriminatoria frente a sus com¿Usted ha asumido alguna vez una pañerossordos?

\begin{tabular}{|l|c|}
\hline SÍ & $10 \%$ \\
\hline NO & $89 \%$ \\
\hline NO RESPONDE & $1 \%$ \\
\hline
\end{tabular}

¿Usted ha evidenciado en algún pañeros sordos? momento discriminación hacia sus com-

\begin{tabular}{|l|c|}
\hline SÍ & $67 \%$ \\
\hline NO & $32 \%$ \\
\hline NO RESPONDE & $1 \%$ \\
\hline
\end{tabular}


Responden padres de hijos con discapacidad visual
¿Su hijo le ha comentado que existe algún tipo de discriminación?

\begin{tabular}{|l|l|}
\hline SÍ & $16 \%$ \\
\hline NO & $67 \%$ \\
\hline NO RESPONDE & $17 \%$ \\
\hline
\end{tabular}

¿Su hijo le ha comentado si existe algún tipo de discriminación en la institución? ¿Cuál?

\begin{tabular}{|c|c|c|c|c|c|c|c|}
\hline & Opciones & Frecuencia & Porcentaje & & Opciones & Frecuencia & Porcentaje \\
\hline \multirow[t]{2}{*}{ Sí } & Malos tratos & 1 & $8 \%$ & \multirow[t]{2}{*}{ NO } & $\begin{array}{l}\text { Mi hijo tiene buena } \\
\text { relación con compañeros } \\
\text { y maestros }\end{array}$ & 1 & $8 \%$ \\
\hline & $\begin{array}{l}\text { En el ámbito } \\
\text { deportivo }\end{array}$ & 1 & $8 \%$ & & No responde & 9 & $75 \%$ \\
\hline
\end{tabular}

Responde: DECE (Departamento de Consejería Estudiantil)

¿Usted ha evidenciado en que los estudiantes sordos han sido víctimas de discriminación en la institución?

\begin{tabular}{|l|l|}
\hline Sí & $33 \%$ \\
\hline N0 & $67 \%$ \\
\hline
\end{tabular}

¿Usted ha evidenciado casos en discriminación en la institución? ¿Cuál? que los estudiantes sordos son víctimas de

\begin{tabular}{|l|l|c|c|c|l|c|c|}
\hline & Opciones & Frecuencia & Porcentaje & & Opciones & Frecuencia & Porcentaje \\
\hline Sí & Agresión físicas & 1 & $33 \%$ & N0 & No responde & 2 & $67 \%$ \\
\hline
\end{tabular}


B. Área: SENSIBILIZACIÓN

Responden: docentes
¿Usted ha participado en talleres o campañas de sensibilización sobre la inclusión educativa de personas sordas?

\begin{tabular}{|l|c|}
\hline SÍ & $57 \%$ \\
\hline NO & $38 \%$ \\
\hline NO RESPONDE & $5 \%$ \\
\hline
\end{tabular}

Responden: Padres de estudiantes sordos

¿La institución educativa ha rea- de sensibilización para que sus hijos sean incluidos en la comunidad educativa?

lizado campañas o algún tipo de talleres

\begin{tabular}{|l|l|}
\hline SÍ & $50 \%$ \\
\hline NO & $50 \%$ \\
\hline
\end{tabular}

Responde: DECE (Departamento de Consejería Estudiantil)

¿En el interior de la institución el sensibilización sobre la inclusión de estudiantes sordos?

DECE ha llevado a efecto campañas de

\begin{tabular}{|l|l|}
\hline SÍ & $67 \%$ \\
\hline NO & $33 \%$ \\
\hline
\end{tabular}

Responden: Estudiantes sordos

¿Considera usted que es necesario realizar trabajos de sensibilización para mejorar su situación en relación con la inclusión educativa en la institución?

\begin{tabular}{|l|l|}
\hline SÍ & $85 \%$ \\
\hline N0 & $15 \%$ \\
\hline
\end{tabular}

Si se trabajara en temas de sensibilización sobre la inclusión educativa, ¿conpara que usted sea un profesional en el sidera que esto generaría un gran aporte 


\begin{tabular}{|l|c|}
\hline SÍ & $100 \%$ \\
\hline NO & $0 \%$ \\
\hline
\end{tabular}

Responden: Padres de estudiantes sordos

¿Cree usted que la puesta en marcha de un programa de sensibilización para los estudiantes en general por medio de cuentos relacionados con el tema de la inclusión educativa, será una contribución para lograr una mejor concientización sobre la condiciones de inclusión de sus hijos?

\begin{tabular}{|l|c|}
\hline Sí & $92 \%$ \\
\hline NO & $8 \%$ \\
\hline
\end{tabular}

C. Área: INCLUSIÓN SOCIAL

Responden: Estudiantes oyentes

¿Se ha mostrado sensible con sus compañeros sordos con la finalidad de colaborar para cumplir alguna actividad ordenada por el docente o se mantiene al margen?

\begin{tabular}{|l|c|}
\hline COLABORO & $10 \%$ \\
\hline ME MANTENGO AL MARGEN & $89 \%$ \\
\hline NO RESPONDE & $1 \%$ \\
\hline
\end{tabular}

¿Sus profesores logran transmitir fomentando la inclusión con sus comun clima acogedor en el aula de clase, pañeros?

\begin{tabular}{|l|l|}
\hline Sí & $85 \%$ \\
\hline N0 & $15 \%$ \\
\hline
\end{tabular}

¿Durante el tiempo de permanen- de discriminación hacia usted o sus comcia en la institución, ha sentido algún tipo pañeros sordos?

\begin{tabular}{|l|l|}
\hline Sí & $10 \%$ \\
\hline N0 & $89 \%$ \\
\hline
\end{tabular}


De los estudiantes oyentes que respondieron Sí, el 31\% consideran que los estudiantes sordos son discriminados por los docentes en vista de que no prestan atención a sus necesidades.

\section{Responden: docentes}

¿Durante las actividades extracurriculares los estudiantes sordos comparten con sus pares oyentes en igualdad de condiciones?

\begin{tabular}{|l|l|}
\hline SÍ & $71 \%$ \\
\hline NO & $19 \%$ \\
\hline NO RESPONDE & $10 \%$ \\
\hline
\end{tabular}

¿Considera que los estudiantes mente en la institución? sordos se encuentran incluidos social-

\begin{tabular}{|l|l|}
\hline SÍ & $76 \%$ \\
\hline NO & $14 \%$ \\
\hline NO RESPONDE & $10 \%$ \\
\hline
\end{tabular}

Responden: Padres de estudiantes sordos

¿Necesita y encuentra colabora- ción por parte de sus compañeros con relación al cumplimiento de determinadas actividades?

\begin{tabular}{|l|c|}
\hline SÍ & $77 \%$ \\
\hline NO & $15 \%$ \\
\hline NO RESPONDE & $8 \%$ \\
\hline
\end{tabular}

¿Cómo es la relación que tiene con sus compañeros oyentes?

\begin{tabular}{|l|c|}
\hline EXCELENTE & $67 \%$ \\
\hline BUENA & $31 \%$ \\
\hline REGULAR & $8 \%$ \\
\hline MALA & $0 \%$ \\
\hline
\end{tabular}


D. Área: RECURSOS EDUCATIVOS

Responden: docentes
¿La institución le facilita las herramientas necesarias para trabajar con los estudiantes sordos?

\begin{tabular}{|l|l|}
\hline Sí & $24 \%$ \\
\hline N0 & $76 \%$ \\
\hline
\end{tabular}

¿Qué tipo de recursos considera procesos de inclusión educativa de estuque son beneficiosos para trabajar en los diantes sordos?

\begin{tabular}{|c|c|}
\hline $\begin{array}{c}\text { Recursos } \\
\text { visuales }\end{array}$ & $81 \%$ \\
\hline
\end{tabular}

¿Usted, docente que trabaja en hace falta algo a la institución? ¿Qué inclusión educativa, considera que para hace falta? una educación de calidad y equidad le

\begin{tabular}{|l|l|}
\hline $\begin{array}{l}\text { Capacitaciones y recur- } \\
\text { sos audiovisuales para } \\
\text { estudiantes sordos }\end{array}$ & $100 \%$ \\
\hline
\end{tabular}

\section{E. Área: CAPACITACIÓN}

Responden: docentes
¿Ha recibido algún tipo de capacitación para trabajar con estudiantes sordos?

\begin{tabular}{|l|l|}
\hline Sí & $71 \%$ \\
\hline N0 & $29 \%$ \\
\hline
\end{tabular}

El 10\% de docentes señala que recibió capacitación únicamente cuando los estudiantes ingresaron, la capacitación no ha sido continua.
¿Considera que las autoridades institucionales aportan en el proceso de inclusión educativa? 


\begin{tabular}{|l|c|}
\hline SÍ & $48 \%$ \\
\hline NO & $43 \%$ \\
\hline NO RESPONDE & $9 \%$ \\
\hline
\end{tabular}

Responde: DECE (Departamento de Consejería Estudiantil) ¿Cuál sería, según su criterio, la gio? principal barrera para la inclusión educativa de estudiantes sordos en el Cole-

\begin{tabular}{|l|l|}
\hline $\begin{array}{l}\text { Falta de capacitación } \\
\text { al personal docente }\end{array}$ & $100 \%$ \\
\hline
\end{tabular}

Otros resultados significativos en esta investigación corresponden a las narrativas de los estudiantes sordos. Al consultarles acerca del proceso de inclusión, encontramos las siguientes respuestas:

- "Le falta mucho al proceso. Las personas sordas somos visuales. Falta mucho apoyo de las autoridades".

- "Primero el programa de inclusión es muy bueno, pero falta mucho, mucho para que se desarrolle de mejor manera".

En respuesta a las necesidades requeridas en relación a los recursos educativos para mejorar los procesos, los participantes refieren lo siguiente:

- "Siento que faltan recursos de tecnología del colegio, como el infocus para entender las clases".
"Siento que hacen mucha falta los recursos visuales, porque así podremos aprender mejor. También las intérpretes son buenas, ellas deberían enseñar lengua de señas básicas a todos los docentes para que no haya discriminación y una buena comunicación".

- "Falta que los profesores estudien lengua de señas básicas. Somos visuales, necesitamos proyectores e imágenes para aprender de mejor manera".

En lo que respecta a la capacitación de docentes, sensibilización y discriminación, los estudiantes sordos refieren:

- "Los profesores sí enseñan bien, pero creo que deben aprender a comunicarse más con los sordos". 
- "En el caso de los maestros, ellos prestan mucha más atención a los oyentes, porque no saben lengua de señas cuando yo necesito algo no me ayudan, deberían seguir aprendiendo más lengua de señas. Quisiera que la clase sea más activa, a veces los profesores hablan muchoy me siento muy aburrida".

- "Creo que los profesores pueden ayudar a los sordos mucho, pero necesitan aprender lengua de señas y dar mejor las clases".

- "Hace falta sensibilización: que la gente comprenda cómo nos sentimos y que puedan conocer más de la cultura sorda y que todos conozcan como es el trabajo de la inclusión que se trata de la igualdad y tener muchas ideas cumpliendo también los deberes".

- "Falta mucha sensibilización, porque hay un poco de discriminación más en un caso con alguna intérprete tuve problemas. Me sentía solo, me sentía discriminado, y sentía que me trataba mal, pero solo puedo hablar con ella, con mi intérprete".

Las narrativas más significativas por parte de los docentes se presentan a continuación:

- "Pienso que la educación inclusiva en el colegio se está llevando de buena manera, pero sí se debe tener en cuenta que no es para todos los estudiantes puesto que, si bien es cierto, pueden desarrollar algunas potencialidades, pero sí es bueno que los que necesitan apoyo extra sí se encuentren en una institución de apoyo especial".

- "Los estudiantes sordos que se encuentran incluidos en la institución se llevan bien entre sí, a pesar de que su relación con los compañeros oyentes no es mejor puesto que existe una barrera en la comunicación, lo cual se evidencia al momento de las actividades deportivas o en el recreo".

"La falta de recursos de trabajo, como los infocus propios, es algo que está retrasando el programa de inclusión, puesto que hay ocasiones que yo, como profesora, debo traer los proyectores de mi domicilio para poder realizar una clase más inclusiva".

"La sensibilización sí hace falta, podría ayudar a muchos a entender las cosas que los estudiantes sordos sienten, pero sería mucho mejor poder crear más ambiente de participación directa entre compañeros, formando campañas de sensibilización para todos, no solo para los sordos que son menos que los oyentes, quienes de igual modo tie- 
nen conflictos. Podría ser producti-

litudes que tienen los estudiantes" vo al visualizar las diferencias y simi-

\section{DISCUSIÓN Y CONCLUSIONES}

Resulta significativo que un $85 \%$ de los estudiantes sordos respondan que ha sufrido algún tipo de discriminación. Es un valor extremadamente alto si se toma en cuenta que estos estudiantes están precisamente inmersos en un proceso de inclusión. Un porcentaje así desdice de los procesos de inclusión educativa y exige, desde ya, analizar a profundidad los resultados que en esta y las otras áreas se presentarán.

En este sentido, el mayor porcentaje de quienes consideran que han sufrido algún tipo de discriminación, señalan que esa discriminación ocurre por falta de atención de los docentes, es decir, precisamente en el espacio y con uno de los actores principales del proceso de inclusión educativa. En segundo lugar, expresan que es la falta de atención en el bar, es decir, un espacio de inclusión social.

El que la falta de atención de los docentes sea el principal tipo de discriminación está relacionado con el porcentaje que ubica precisamente a los docentes como los más importantes actores de esa discriminación. Le siguen, porcentualmente, las autoridades y los compañeros.
Llama la atención que solo el 16\% de los padres de los estudiantes sordos señalen que su hijo comunicó un tipo de discriminación -en abierta contraposición a la cantidad de estudiantes sordos que consideran haber sufrido algún tipo de discriminación-. Marca también un contraste el que el 33\% de los psicólogos del DECE afirme haber conocido de casos de discriminación.

Podemos inferir de estos resultados que existe una falta de comunicación entre padres e hijos con discapacidad auditiva. Únicamente el 16\% comenta que sus hijos han sufrido discriminación mientras que el $85 \%$ de sus hijos mencionan que sí son discriminados.

En el área de sensibilización, es evidente que no todo el personal docente ha pasado por un proceso pertinente para incluir a los estudiantes sordos. Lo mismo ocurre con la mayoría de los padres de familia. En este aspecto, se debe tomar en cuenta que todos los participantes han expresado la necesidad de realizar procesos de sensibilización y consideran este aspecto como uno de los primordiales para lograr una inclusión eficaz. Uno de los participantes hace re- 
ferencia específicamente a la sensibilización con miras a la comprensión acerca de los sentimientos de las personas sordas y al conocimiento de la cultura sorda.

Si bien es cierto que el DECE y las autoridades afirman que se han realizado campañas de sensibilización, no se ha llegado a la totalidad de la comunidad educativa. Sería importante, además, analizar la forma en que se han realizado estas campañas, si son parte del proyecto de inclusión institucional, si han sido planificadas y ejecutadas desde las necesidades institucionales.

Por otro lado, el tema de la capacitación docente requiere de un profundo análisis, pues resulta preocupante que únicamente el 10\% de los docentes haya recibido capacitación durante el inicio del proceso, es decir, que la formación no ha sido sostenida en el tiempo a pesar de que el personal del DECE menciona que la falta de capacitación docente es la principal barrera para la inclusión de los estudiantes sordos. Estos, por su parte, también expresan la necesidad de capacitación para los docentes. Resulta interesante considerar que la capacitación a la que se refieren tiene que ver con el aprendizaje de lengua de señas. Los estudiantes sordos afirman que los docentes prestan más atención a los estudiantes oyentes debido a la barrera de la comunicación. El desconocimiento total en lengua de señas ocasiona que los estudiantes se sientan desatendidos y de cierta forma discriminados. Sería importante considerar la posibilidad de capacitar a los docentes al menos en el aprendizaje de palabras básicas de manera que la comunicación y la atención puedan mejorar.

En relación a este punto podemos hacer referencia al Reglamento de Régimen Académico, el cual fue aprobado por el Consejo de Educación Superior (CES) el 21 de noviembre de 2013 y entró en vigencia a partir del 28 del mismo mes, donde se establece que las asignaturas destinadas a los aprendizajes de la lengua extranjera garantizarán el nivel de suficiencia del idioma para cumplir con el requisito de graduación de las carreras de tercer nivel (Ley Orgánica de Educación Superior, 2013). De este modo se podría pensar en la posibilidad de que la lengua de señas pueda ser considerada como un segundo idioma, así los estudiantes de carreras pedagógicas podrían elegir en lugar del inglés o el italiano, la lengua de señas como segundo idioma para lograr adquirir conocimientos que les permita lograr una adecuada comunicación con estudiantes que presenten sordera, lo que mejoraría los procesos de inclusión educativa.

Otras investigaciones también han analizado el tema de la falta de conocimiento de la lengua de señas. Este es uno de los resultados obtenidos en la in- 
vestigación titulada Educación para la inclusión de estudiantes sordos de la Revista Latinoamericana de Educación Inclusiva:

Los problemas que señalan se sitúan en la falta de profesores que conozcan y dominen la lengua de signos y la utilicen de forma efectiva en los procesos de enseñanza-aprendizaje de estos alumnos; en las dificultades para interactuar con los alumnos oyentes y con los profesores al no compartir un código comunicativo; así como en las dificultades para seguir el ritmo de aprendizaje de sus compañeros oyentes de aula (Domínguez, 2009, p. 46).

Además, es vital que las instituciones educativas realicen procesos de evaluación periódicos con miras a que cada escuela logre mejorar en función de sus circunstancias y sus posibilidades. Eso es lo que hay que garantizar: el avance sin pausa de la calidad educativa que llega al conjunto de la población (Casanova, 2017).

Podemos concluir que la sensibilización dentro de la institución educativa no se ha trabajado significativamente. Es una necesidad de los estudiantes que requiere ser atendida continuamente en la institución, principalmente en las aulas de clase. Se requiere incrementar los procesos de sensibilización dentro de la institución debido a que existe un 85\% de estudiantes sordos que se sienten discriminados principalmente por los docentes, lo que provoca malestar en su permanencia en la institución, y ha afectado su desarrollo emocional así como ha disminuido su autoestima. El desarrollo emocional recepta elementos desde el mundo externo para constituirse desde lo propio e impactar en el desarrollo humano. (Mulsow, 2008).

El $95 \%$ de docentes y el $100 \%$ de estudiantes oyentes están de acuerdo con que los estudiantes sordos estén incluidos en la institución, porque conocen que todos tienen los mismos derechos y obligaciones. Sin embargo, existe un $24 \%$ de estudiantes oyentes que considera que la inclusión interfiere con el desarrollo de su aprendizaje y un 5\% de docentes que piensa que los estudiantes sordos deberían tener una educación especial. En este sentido hay que resaltar que el Reglamento General de la Ley Orgánica de educación Intercultural señala la atención prioritaria que debe brindarse a los estudiantes que presentan necesidades educativas especiales asociadas a la discapacidad lo que hace referencia a los procesos de inclusión que deben llevarse a cabo en instituciones educativas regulares con las adaptaciones requeridas. (Constitución del Ecuador, 2012). Las necesidades educativas especiales son las condiciones que afectan el 
desarrollo personal del estudiante y que justifican la provisión de determinadas ayudas (Bermeosolo, 2010).

Los resultados ratifican que no existe una adecuada capacitación de los docentes para trabajar en inclusión. Esto se ha convertido en una barrera para los estudiantes sordos en su proceso inclusión educativa. Es necesario implicarse en la atención de aquellos que consideramos diferentes para que podamos dejar de verlos diferentes (Chávez, Cúpich, \& Alvarado, 2016).

En relación con los padres de familia, el conocimiento acerca del proceso de inclusión educativa es escaso y no se ha brindado por canales oficiales de la institución. No han sido informados, por tanto, de manera específica sobre el programa de inclusión y no se les han entregado informes de seguimiento del desarrollo de sus hijos dentro del programa.

En lo referente a los recursos materiales, la institución no cuenta con los recursos digitales que permitan llevar a cabo de manera eficiente el programa de inclusión. Esto se ha evidenciado puesto que los estudiantes sordos no cuentan con las herramientas visuales necesarias para su adecuada accesibilidad académica y de comunicación.

En el aspecto de la inclusión social, se concluye que un 15\% de los estudiantes sordos no ha sido incluido socialmente por parte de los estudiantes oyentes, esto se debe a que no se realizan actividades con miras a la inclusión social de este grupo de estudiantes.

Las recomendaciones que surgen a partir de esta investigación se presentan a continuación.

- Trabajar en procesos de inclusión educativa en el marco de los principios de normalización, integración e individualización, considerando las características, intereses, capacidades y necesidades propios de cada estudiante.

- Continuar construyendo recursos psicoeducativos innovadores basados en las experiencias de las personas sordas, siempre a partir del principio de individualización. Los recursos que se presenten dentro de inclusión deben estar referidos directamente a las necesidades y experiencias de las personas con discapacidad.

- Los procesos de sensibilización deben ser considerados como un punto de trabajo primordial dentro de las instituciones educativas.

- Se deben generar espacios informativos de sensibilización dentro del programa de inclusión educativa, para que de este modo se reduzca la discriminación en la institución y los estudiantes sordos puedan mejorar su autoestima. 
- Incrementar los procesos de sensibilización con el objetivo de lograr que el 100\% de los docentes acepten el programa de inclusión en la institución, de igual modo realizar capacitaciones y campañas informativas sobre la importancia de la inclusión educativa para la comunidad, de modo que se generen espacios en los que se expresen ideas que lleven a acuerdos positivos sobre la inclusión educativa.

- Resulta fundamental capacitar a los docentes para que esto refleje una real inclusión en la institución, a través de la concientización sobre la importancia de que los docentes aprendan un nivel mínimo de la lengua de señas para comunicarse y atender las necesidades de sus estudiantes personalmente.

- Crear un protocolo de comunicación para padres de familia, en el cual periódicamente estos puedan informarse sobre el desarrollo de sus hijos con respecto al programa de inclusión.

- La institución debe generar medios de autogestión, los cuales les facilitarán la adquisición de materiales adecuados para trabajar en inclusión educativa, además es necesario obtener herramientas visuales propias de la institución, estas contribuirán en el aprendizaje significativo de los estudiantes.
- El DECE de la institución debe trabajar en generar sensibilización y colaboración por parte de los estudiantes oyentes hacia los estudiantes sordos a través de un espacio en que los estudiantes oyentes aprendan lengua de señas, de esta manera se atenderá a las necesidades sociales dentro del programa de inclusión.

Finalmente, se debe mencionar que en este artículo no se hace uso del término "discapacidad auditiva". En su lugar, hemos empleado el término "sordo" debido a que las personas sordas pertenecen a la comunidad sorda donde la lengua de señas ecuatoriana les otorga identidad, pues, a través de ella, se consideran una comunidad lingüística minoritaria. Esto nos invita a sostener una visión socioantropológica y no limitarnos a una mirada física -biológica- médica. El ser humano es mucho más que un oído.

Esta investigación aporta con información significativa adquirida en un proceso de investigación que revela la realidad particular del proceso de inclusión educativa en un centro educativo público con lo que brinda un panorama actual de lo que ocurre en este campo en la educación ecuatoriana. Nuestra limitante fue el desconocimiento en lengua de señas, la participación de los estudiantes sordos se la realizó con el apoyo de intérpretes técnicos y califica- 
dos. Sin embargo, sentimos la necesidad de aprender a comunicarnos con las personas sordas. A partir de esta investigación proponemos estudios futuros encaminados a la inclusión laboral de personas sordas, así como las necesidades de capacitación en los docentes ecuatorianos en temas de atención a estudiantes con necesidades educativas especiales y el análisis de las mallas curriculares de instituciones de educación superior en el área de las Ciencias de la Educación y la Psicología Educativa. 


\section{REFERENCIAS}

Asamblea Nacional del Ecuador. (2013). Ley Orgánica de Educación Superior. Quito: Corporación de Estudios y Publicaciones.

Bermeosolo, J. (2010). Psicpedagogia de la diversidad en el aula. México: Alfaomega.

Boza Carreño, Á., Méndez Garrido, J. M., Monescillo Palomo, M., \& Toscano Cruz, M. d. (2010). Educación, investigación y desarrollo social. Madrid: Narcea Ediciones.

Casanova, M. A. (2017). Educación inclusiva en las aulas. Madrid: Arco.

Chávez, E., Cúpich, Z., \& Alvarado, J. (2016). Sistema educativo e inclusión. México: Trillas.

Constitución del Ecuador. (2012). Ley Orgánica de educación Intercultural. Quito: Corporación de estudios y pcublicaciones.

Domínguez, A. B. (2009). Educación para la inclusión de alumnos sordos. Revista Latinoamericana de Educación Inclusiva, 46.
Echeita, G. (2008). INCLUSIÓN Y EXCLUSIÓN EDUCATIVA. REICE - Revista Electrónica Iberoamericana sobre Calidad, Eficacia y Cambio en Educación, 918.

Kaplún, G. (2005). Contenidos, itinerarios y juegos. Revista Interamericana de Educación de Adultos, 143158.

López Cortiñas, C. (2005). Prólogo. In M. d. Puelles Benítez, Educación, igualdad y diversidad cultural ( $p$. 911). Madrid: Biblioteca Nueva .

Marcelo García, C., \& Vaillant, D. (2009). Desarrollo profesional docente: ¿cómo se aprende a enseñar? Madrid: Narcea Ediciones.

Ministerio de Educación del Ecuador. (2011). programa de sensibilización. Retrieved from Ministerio de Edcuación: https://educacion. gob.ec

Mulsow, G. (2008). Desarrollo emocional: Impacto en el desarrollo humano. Educacao, 63. 